\title{
Determinants of Social Insurance Coverage in the Philippines
}

\author{
Ma. Belinda S. Mandigma
}

\begin{abstract}
The study analyzed the coverage of the most dominant form of social security which is social insurance or public pension in the Philippines by examining the relationships with selected key economic and non-economic variables. Aggregated panel data for the period 2000-2013 were tested using Generalized Linear Model, Multivariate Ordinary Least Squares, Fixed effects \& Random Effects and Generalized Method of Moments. Results revealed that coverage represented by contributors from the economically active population are influenced only by some economic variables like economic stability, capital formation, wage growth and urbanization, economic development and globalization. Coverage corresponding to the elderly beneficiaries is also affected by the foregoing economic factors except for globalization, but inclusive of national administrative capacity, employment and unemployment. Further, non-economic drivers of elderly coverage are feminist theory, education, informal sectors and poverty incidence. More statistically significant determinants for the elderly confirms the conclusion of Rofman and Oliveri (2012) that assessing coverage among the elderly presents fewer difficulties, since instead of measuring the accumulation of potential rights, the focus is on the proportion of eligible individuals who are currently receiving benefits.
\end{abstract}

Index Terms-Economically active population, elderly beneficiaries, social insurance coverage, social security.

\section{INTRODUCTION}

The international labor office [1] defines social security as "the protection which society provides for its members through a series of public measures namely: social insurance, social assistance and social allowance. It should be noted, however, that social insurance is the most dominant form of social security [2]. Further, King [3] maintained that social security is a defined-benefit pension plan managed by the government by collecting "contributions" in the form of social security taxes and paying benefits according to formulas set by Congress. Thus, in this context, social security may be interpreted to be interchangeable with welfare state. According to Oxford dictionary, welfare state is a system whereby the government undertakes to protect the health and well-being of its citizens, especially those in financial or social need. A basic feature of welfare state is social insurance, intended to provide benefits during periods of greatest need (e.g., old age, illness, unemployment).

The performance of social security systems around the

Manuscript received April 23, 2015; revised July 3, 2015.

Ma. Belinda S. Mandigma is with the Research Center for Culture, Education and Social Issues, the Graduate School and the College of Commerce of the University of Santo Tomas, Manila, Philippines (e-mail: belmandigma@yahoo.com). world can usually be evaluated considering three particular dimensions, namely: coverage, adequacy and sustainability [4]. However, social security systems have been under challenge pointing to deficiencies in the level of protection and the scope of coverage [5].

With fifteen different pension schemes, the Philippines has the highest number of pension schemes in East and Southeast Asia [6]. However, in terms of coverage, the country has one of the lowest in the region. At this juncture, it is timely to examine how to enhance social security coverage in the Philippines, both legally and effectively. The objective of this study is to analyze the coverage of the most dominant form of social security, social insurance or public pension of the Social Security System and the Government Social Insurance System in the Philippines by examining their relationships with selected key economic and non-economic factors.

This study aims to contribute to the literature with up-to-date perspective of public pension coverage in the Philippines and bring it a bit closer to similar studies designed to give an overall view of social insurance coverage in the world.

\section{LITERATURE REVIEW}

Social Security coverage is a multi-dimensional concept which can be defined either as legal coverage or effective coverage [7]. A population group can be identified as legally covered if there are existing legal provisions that such a group should be covered by social insurance by and for a given branch of social security, or will be entitled to specific benefits under certain circumstances - for instance, to an old age state pension on reaching the age of 65 , or to income support if income falls below a specified threshold, or to national health services when sick. On the other hand, effective coverage is measured, for example, by the number of people actually contributing to social insurance in a given branch, or the number of beneficiaries of any pension benefits among all residents over 65 years of age, or the number of beneficiaries of some kind of income support among all those unemployed or all below the poverty line. Rofman and Oliveri [7] proposed to distinguish between contributors (those that contribute to the system in a given moment) and members or affiliates (those that at some time registered in the system, regardless of whether they continued their contributions) and learned that the ratio of contributors to employed workers (occupational coverage) or contributors to the economically active population (coverage of the labor force) produces better estimates of coverage.

In the Philippines, there is very low coverage of economically active population (EAP) and the elderly [6]. 
Social insurance coverage in the Philippines is under challenge because $71 \%$ of EAP and $82 \%$ of elderly are not covered. Several reasons were hypothesized to have caused this low coverage, e. g., large informal sector ( $42 \%$ of EAP), huge poverty incidence $(26.5 \%)$, high unemployment $(7.5 \%)$ and underemployment $(19.4 \%)$ rates. Surprisingly, the global crisis did not reduce overall coverage.

In terms of empirical work on the drivers of social insurance coverage in different countries, several authors examined some possible variables. Corripio [8] assessed the factors for voluntary contribution to pension funds by independent workers in Chile including monetary value of durable and capital goods, years of education, number of children in the house, type of work whether permanent, fixed term or temporary, and found that workers will have greater incentive to save if given access to education, health services and housing. The study's findings complement those of Levy [9]; Galiani and Weinschelbaum [10]; and Kitao [11]. Hong [12] examined the trends and determinants of social expenditure in Korea, Japan and Taiwan using the following variables: public social expenditure as percent of GDP, unemployment as percent of total labor force, annual growth rate of GDP, employment in agriculture as percent of total employment, general government final consumption expenditure as \% of GDP, trade as percent of GDP and global crisis. However, results suggest that political determinants would probably represent the most important field for welfare development, due perhaps to the contention of Kim [13] that grouping subject Asian countries with some incoherent features among them would be a problem. This is in contrast to the findings of the following authors with regards to the following influential variables: Lin [14] modernization; Kim and Jung [15] modernization, globalization and power resources; Ahn and Baek [16], Rupp and Stapleton [17] feminist and market theories; Ahn and Lee [18] economic development and modernization; Park [19] effects of globalization and economic crisis; Croisant [20] demographic, economic and political trends; and Cristea et al. [21] economic growth. Kaseke [2] studied the challenges of extending social security to the poor in an African perspective and found that one of the reasons why the coverage of formal social security systems is low is because the self-employed domestic workers and those employed in the informal sector are not participating in the scheme. Rickne [22] studied the relationship between labor market conditions and social insurance participation among Chinese industrial firms and found that the increased scarcity of labor, large shares of uninsured workers, large shares of low-educated workers and those without labor unions were quantitatively important drivers of participation. The results of his tests were supported by the findings of the studies by different authors, namely: Cai [23], Meng [24], and Blechova et al. [25] impact of aging population and rural-urban migration; Lu, Tao and Wang [26] unionization; Nyland et al. [27]; Nielsen and Smith [28] negative association of firm size; Mao et al. [29] positive association of firm size; Nyland et al. [30], Cheng et al. [31], Guo and Gao [32], Smyth et al. [33] education impact; Nielsen et al. [34], Smyth et al. [33] urban residence permit; Gao et al. [35] labor contract status.

A number of studies analyzed the determinants of coverage by comparing data from several countries. Auerbach et al. [36] found that the self-employed are indistinguishable from salaried workers in deciding about contributing to a pension program in developing countries. Khan et al. [37] examined annual data from 13 OECD member countries using the following explanatory variables: country unemployment rate, per capita GDP, mortality, proportion of female in total population, proportion of people living in urban areas to total population, proportion of people aged 55-65 years participating in labor market, age of social security system. Inclusion of mortality rate as an additional variable in the analysis did not affect the findings that a decline in unemployment increased sickness benefits spending and reduced disability pension spending. Turner [38] studied the retirement income system for different economic, demographic and political environment (166 countries) and found that the level of economic development, the population age structure and political factors affect the retirement income system appropriate for different countries. Aspalter [39] predicted a convergence of social welfare policies based on common structural determinants such as the degree of economic development, urbanization, modernization and the advance of capitalism. Other studies supporting the findings of the foregoing research are those of $\mathrm{Ku}$ and Finer [40] and of Jensen [41]. The ILO study of 2001 showed that worldwide, probably not more than a quarter of the $150 \mathrm{M}$ unemployed people are covered by unemployment benefits and that they are mainly concentrated in industrial countries. Further, it also found that globalization often exposes societies to greater income insecurity.

The current study will enrich the existing literature on the determinants of social insurance coverage. The factors that can impact pension coverage are explained in the next section.

\section{MODEL FORMULATION}

\section{A. The Oretical Framework}

Since social insurance is a basic feature of welfare state, this study will use the explanatory or analytical theory serving as a testing ground for welfare systems in East Asia. In the face of the numerous possible determinants and impact factors that has been identified in the past, the business of welfare state is vital to identify the permanent interplay of different causes and underlying or side factors [39].

\section{B. Conceptual Framework}

Social insurance coverage is affected by both economic and non-economic factors. From the economic perspective, public pension program participation is an outcome of the interaction of the "demand" for the program by individuals and the "supply" of program benefits by employers through the government. Meanwhile, demographic, social and structural factors, at least in a proximate sense, are non-economic variables that might affect public pension coverage. In this study, coverage is measured considering the percentage of the economically active population (EAP) that contribute to the pension system as the main coverage indicator among the EAP and the percentage of population 60 years or more that receives pension benefits as the main coverage indicator among the elderly. Table I presents the key factors identified to affect social insurance coverage. 
TABLE I: MAJOR FACTORS THAT AFFECT SOCIAL INSURANCE COVERAGE

\begin{tabular}{|c|c|c|}
\hline Factors & Operationalization & Expected Relationship \\
\hline \multicolumn{3}{|l|}{ Economic } \\
\hline $\begin{array}{l}\text { Degree of Economic } \\
\text { Development }\end{array}$ & $\begin{array}{l}\text { PPP GDP per Capita } \\
\text { growth rate }\end{array}$ & $\begin{array}{l}\text { Positive with higher } \\
\text { development stage }\end{array}$ \\
\hline Modernization & $\begin{array}{l}\text { Proxy is annual growth } \\
\text { rate of GDP at constant } \\
2000 \text { prices }\end{array}$ & $\begin{array}{l}\text { Positive with higher } \\
\text { degree of modernization }\end{array}$ \\
\hline Economic Stability & Proxy is inflation rate & $\begin{array}{l}\text { (Reverse) Positive } \\
\text { w/lower rates }\end{array}$ \\
\hline Capital Formation & $\begin{array}{l}\text { Capital formation growth } \\
\text { rate at constant } 2000 \\
\text { prices }\end{array}$ & $\begin{array}{l}\text { Positive with higher } \\
\text { rates }\end{array}$ \\
\hline $\begin{array}{l}\text { National } \\
\text { Administrative } \\
\text { Capacity }\end{array}$ & $\begin{array}{l}\text { Consolidated public } \\
\text { sector financial position } \\
\text { (surplus/deficit) as \% of } \\
\text { GDP }\end{array}$ & $\begin{array}{l}\text { Positive with higher } \\
\text { rates }\end{array}$ \\
\hline Globalization & $\begin{array}{l}\text { Overall BOP position } \\
\text { as } \% \text { of GDP }\end{array}$ & $\begin{array}{l}\text { Negative as it exposes } \\
\text { societies to greater } \\
\text { income insecurity }\end{array}$ \\
\hline Global Crisis & $\begin{array}{l}\text { Dummy variable } \\
\text { represented by " } 1 \text { " for } \\
2008-10 \text {; "0" for } 2000-07 \\
\& 2011-13\end{array}$ & $\begin{array}{l}\text { Negative as it reduces } \\
\text { income security \& social } \\
\text { protection }\end{array}$ \\
\hline Wage Growth Rate & Wage share in GDP (\%) & $\begin{array}{l}\text { Positive with higher } \\
\text { rates }\end{array}$ \\
\hline Unemployment & Unemployment rate & $\begin{array}{l}\text { Negative with increased } \\
\text { rate }\end{array}$ \\
\hline Under employment & Underemployment rate & $\begin{array}{l}\text { Negative with increased } \\
\text { rate }\end{array}$ \\
\hline Urbanization & $\begin{array}{l}\text { Employment in } \\
\text { Agriculture growth rate }\end{array}$ & $\begin{array}{l}\text { (Reverse) Positive with } \\
\text { lower \% }\end{array}$ \\
\hline \multicolumn{3}{|l|}{ Non-economic } \\
\hline Union Density Rate & Trade Unions & $\begin{array}{l}\text { Positive with more } \\
\text { unions }\end{array}$ \\
\hline $\begin{array}{l}\text { Social Security } \\
\text { Benefits }\end{array}$ & $\begin{array}{l}\text { Social security benefits } \\
\text { as } \% \text { of GDP }\end{array}$ & $\begin{array}{l}\text { Positive as additional } \\
\text { benefits induce higher } \\
\text { contributions }\end{array}$ \\
\hline Informal Sectors & $\begin{array}{l}\text { Self-employed \& Unpaid } \\
\text { family workers \% to total } \\
\text { employment }\end{array}$ & $\begin{array}{l}\text { Negative with large } \\
\text { informal sectors }\end{array}$ \\
\hline Feminist Theory & $\begin{array}{l}\text { Female share of } \\
\text { employment }\end{array}$ & $\begin{array}{l}\text { Positive with higher } \\
\text { share }\end{array}$ \\
\hline Family Obligation & $\begin{array}{l}\text { Economically inactive } \\
\text { due to household/ family } \\
\text { duties as \% of working } \\
\text { age populace }\end{array}$ & Negative with higher $\%$ \\
\hline Education & $\begin{array}{l}\text { Adult secondary grad } \\
\text { rate, } 25+\text { years old }\end{array}$ & $\begin{array}{l}\text { Positive with higher } \\
\text { rates }\end{array}$ \\
\hline Poverty Incidence & $\begin{array}{l}\text { Poverty incidence per } \\
\text { population }\end{array}$ & $\begin{array}{l}\text { Negative w/ increased } \\
\text { poverty }\end{array}$ \\
\hline
\end{tabular}

\section{Equation Models}

In view of the foregoing, the following models were formulated:

$$
\begin{aligned}
\mathrm{SIC}_{\mathrm{EAP}} & =\alpha+\sum \beta X_{e+} \varepsilon \\
\mathrm{SIC}_{\mathrm{EAP}} & =\alpha+\sum \beta X_{n e+} \varepsilon \\
\mathrm{SIC}_{60+} & =\alpha+\sum \beta X_{e+} \varepsilon \\
\mathrm{SIC}_{60+}= & \alpha+\sum \beta X_{n e+} \varepsilon
\end{aligned}
$$

where the percentage of EAP that contributes to the pension system will proxy for the degree of social insurance coverage (dependent variable) among the economically active population $\left(\mathrm{SIC}_{\mathrm{EAP}}\right)$ while the percentage of population 60 years or more that receives pension benefits will proxy for the degree of social insurance coverage (dependent variable) among the elderly $\left(\mathrm{SIC}_{60+}\right) . X_{e}$ is a set of independent economic variables identified to be the potential determinants of social insurance coverage while $X_{n e}$ is a set of independent non-economic factors also identified to be potential drivers of social insurance coverage as presented in Table I.

\section{MethodS}

The equation models highlighted in the foregoing discussions were tested using multiple regression models based on panel data for the period 2000- 2013 at an annual frequency. The software Stata12 was used for the statistical treatment of data. The panel data for both the dependent and independent variables were sourced from the Decent Work Statistics (DEWS) - Philippines and the Bangko Sentral ng Pilipinas (BSP).The multiple regression models used to examine the relationship between social insurance coverage and the different economic and non-economic factors are the following:

1) Generalized Linear model (GLM),

2) Multivariate Ordinary Least Squares (OLS),

3) Fixed Effect (FE) and Random Effect (RE) and

4) Generalized Method of Moments (GMM).

\section{RESULTS}

The results of the GLM and multivariate OLS regressions with economic variables are given in Table II while those with non-economic variables are in Table III. Note that the coefficients for these tests are identical but with different

\begin{tabular}{|c|c|c|c|c|c|c|}
\hline \multirow{3}{*}{$\begin{array}{l}\text { Economic } \\
\text { Variables }\end{array}$} & \multicolumn{3}{|c|}{$\begin{array}{c}\text { Economically Active } \\
\text { Population }\end{array}$} & \multicolumn{3}{|c|}{ Sixty Plus Years Old } \\
\hline & \multirow[t]{2}{*}{ Coefficient } & \multicolumn{2}{|c|}{ t Standard Errors } & \multirow[t]{2}{*}{ Coefficient } & \multicolumn{2}{|c|}{ Standard Errors } \\
\hline & & GLM & OLS & & GLM & OLS \\
\hline $\begin{array}{l}\text { Economic } \\
\text { Development }\end{array}$ & 27.89 & $13.89 * *$ & 13.89 & -21.57 & $4.620 * * *$ & $4.620 * *$ \\
\hline Modernization & -27.75 & $13.70 * *$ & 13.7 & 21.38 & $4.557 * * *$ & $4.557^{* *}$ \\
\hline $\begin{array}{l}\text { Economic } \\
\text { Stability }\end{array}$ & 0.396 & 0.574 & 0.574 & -1.144 & $0.191 * * *$ & $0.191 * *$ \\
\hline $\begin{array}{l}\text { Capital } \\
\text { Formation }\end{array}$ & 0.156 & $0.045^{* * *}$ & $0.045^{*}$ & -0.081 & $0.015^{* * *}$ & $0.015^{* *}$ \\
\hline $\begin{array}{l}\text { Administrative } \\
\text { Capacity }\end{array}$ & -0.802 & 0.537 & 0.537 & 1.398 & $0.178 * * *$ & $0.178^{* *}$ \\
\hline Wage Growth & -0.093 & 0.265 & 0.265 & 0.951 & $0.088 * * *$ & $0.088^{*} *$ \\
\hline Globalization & -0.606 & $0.215 * * *$ & 0.215 & 0.09 & 0.071 & 0.071 \\
\hline Global Crisis & -7.276 & $4.103^{*}$ & 4.103 & 8.631 & $1.364 * * *$ & $1.364 * *$ \\
\hline Unemployment & $\mathrm{t}-0.272$ & 0.395 & 0.395 & 0.288 & $0.132 * *$ & 0.132 \\
\hline $\begin{array}{l}\text { Under } \\
\text { employment }\end{array}$ & -0.012 & 0.45 & 0.45 & 0.345 & $0.150 * *$ & 0.15 \\
\hline Urbanization & -0.584 & $0.277 * *$ & 0.277 & 0.419 & $0.092 * * *$ & $0.092^{* *}$ \\
\hline Intercept & 83.53 & $39.16 * *$ & 39.16 & -57.8 & $13.02 * * *$ & $13.02 * *$ \\
\hline R-squared & 0.988 & & & 0.999 & & \\
\hline
\end{tabular}
probabilities.

TABLE II: RESUlTS OF GENERAL LINEAR MODEL AND MULTIVARIATE ORDINARY LEAST SQUARES FOR ECONOMIC VARIABLES

*** significance @ 1\% level, ** significance @ 5\% level, * significance @ 10\% level. 
TABLE III: RESULTS OF GENERAL LINEAR MODEL AND MULTIVARIATE ORDINARY LEAST SQUARES FOR NON-ECONOMIC VARIABLES

\begin{tabular}{|c|c|c|c|c|c|c|}
\hline \multirow{3}{*}{$\begin{array}{c}\text { Non-Economic } \\
\text { Variables }\end{array}$} & \multicolumn{3}{|c|}{$\begin{array}{c}\text { Economically Active } \\
\text { Population }\end{array}$} & \multicolumn{3}{|c|}{ Sixty Plus Years Old } \\
\hline & \multirow[t]{2}{*}{ Coefficient } & \multicolumn{2}{|c|}{ Standard Errors } & \multirow[t]{2}{*}{ Coefficient } & \multicolumn{2}{|c|}{ Standard Errors } \\
\hline & & GLM & OLS & & GLM & OLS \\
\hline Union Density & -0.249 & 0.669 & 0.669 & -0.018 & 0.158 & 0.158 \\
\hline SS Benefits & -1.546 & 3.841 & 3.841 & -0.082 & 0.908 & 0.908 \\
\hline $\begin{array}{l}\text { Feminist } \\
\text { Theory }\end{array}$ & -0.674 & 1.203 & 1.203 & 0.482 & $0.284^{*}$ & 0.284 \\
\hline Education & 0.174 & 0.736 & 0.736 & 0.602 & $0.174 * * *$ & $0.174 * *$ \\
\hline $\begin{array}{l}\text { Informal } \\
\text { Sector }\end{array}$ & -0.313 & 0.508 & 0.508 & -0.218 & $0.120^{*}$ & 0.12 \\
\hline $\begin{array}{l}\text { Family } \\
\text { Obligation }\end{array}$ & -2.057 & $1.114 *$ & 1.114 & 0.43 & 0.263 & 0.263 \\
\hline Poverty & 0.472 & 1.316 & 1.316 & 0.613 & $0.311^{* *}$ & $0.311^{*}$ \\
\hline Intercept & 88.74 & 62.26 & 62.26 & -46.31 & $14.72 * * *$ & $14.72 * *$ \\
\hline R-squared & 0.844 & & & 0.997 & & \\
\hline
\end{tabular}

The FE and the RE methods are for estimating unobserved effects in the model. The fixed effects method eliminates time-invariant unobserved effects before estimation. The random effects model eliminates unobserved effects in the error term, which are uncorrelated with all the explanatory variables. The results are shown in Table IV with economic determinants and in Table $\mathrm{V}$ with non-economic determinants.

TABLE IV: RESULTS OF FIXED EFFECTS AND RANDOM EFFECTS FOR ECONOMIC VARIABLES

\begin{tabular}{|c|c|c|c|c|}
\hline \multirow{2}{*}{$\begin{array}{l}\text { Economic } \\
\text { Variables }\end{array}$} & \multicolumn{2}{|c|}{$\begin{array}{c}\text { Economically Active } \\
\text { Population } \\
\end{array}$} & \multicolumn{2}{|c|}{ Sixty Plus Years Old } \\
\hline & RE & $\mathrm{FE}$ & $\mathrm{RE}$ & $\mathrm{FE}$ \\
\hline $\begin{array}{l}\text { Economic } \\
\text { Development }\end{array}$ & $\begin{array}{l}-0.335 \\
(0.140)^{* *}\end{array}$ & omitted & $\begin{array}{l}0.127 \\
(0.059) * *\end{array}$ & omitted \\
\hline Modernization & omitted & $\begin{array}{l}-0.311 \\
(0.198)\end{array}$ & omitted & $\begin{array}{l}0.117 \\
(0.083)\end{array}$ \\
\hline $\begin{array}{l}\text { Economic } \\
\text { Stability }\end{array}$ & $\begin{array}{l}-0.658 \\
(0.282)^{* *}\end{array}$ & $\begin{array}{l}-0.732 \\
(0.467)\end{array}$ & $\begin{array}{l}-0.284 \\
(0.118)^{* *}\end{array}$ & $\begin{array}{l}-0.256 \\
(0.196)\end{array}$ \\
\hline Capital Formation & $\begin{array}{l}0.083 \\
(0.021)^{* *} \\
*\end{array}$ & $\begin{array}{l}0.086 \\
(0.028)^{*}\end{array}$ & $\begin{array}{l}-0.013 \\
(0.009)\end{array}$ & $\begin{array}{l}-0.014 \\
(0.012)\end{array}$ \\
\hline $\begin{array}{l}\text { Administrative } \\
\text { Capacity }\end{array}$ & $\begin{array}{l}0.121 \\
(0.255)\end{array}$ & $\begin{array}{l}0.129 \\
(0.310)\end{array}$ & $\begin{array}{l}0.651 \\
(0.107)^{* *} \\
*\end{array}$ & $\begin{array}{l}0.648 \\
(0.130)^{* *}\end{array}$ \\
\hline Wage Growth & $\begin{array}{l}0.420 \\
(0.114)^{* *} \\
*\end{array}$ & $\begin{array}{l}0.413 \\
(0.141)^{*}\end{array}$ & $\begin{array}{l}0.633 \\
(0.048)^{* *} \\
*\end{array}$ & $\begin{array}{l}0.636 \\
(0.059)^{* * *} \\
*\end{array}$ \\
\hline Globalization & $\begin{array}{l}-0.325 \\
(0.232)\end{array}$ & $\begin{array}{l}-0.370 \\
(0.340)\end{array}$ & $\begin{array}{l}-0.036 \\
(0.097)\end{array}$ & $\begin{array}{l}-0.019 \\
(0.142)\end{array}$ \\
\hline Global Crisis & $\begin{array}{l}0.338 \\
(0.905)\end{array}$ & $\begin{array}{l}0.568 \\
(1.478)\end{array}$ & $\begin{array}{l}2.567 \\
(0.379) * * \\
*\end{array}$ & $\begin{array}{l}2.477 \\
(0.620)^{*}\end{array}$ \\
\hline Unemployment & $\begin{array}{l}-0.024 \\
(0.440)\end{array}$ & $\begin{array}{l}-0.010 \\
(0.535)\end{array}$ & $\begin{array}{l}0.206 \\
(0.184)\end{array}$ & $\begin{array}{l}0.201 \\
(0.224)\end{array}$ \\
\hline Underemployment & $\begin{array}{l}0.675 \\
(0.366)^{*}\end{array}$ & $\begin{array}{l}0.753 \\
(0.556)\end{array}$ & $\begin{array}{l}-0.063 \\
(0.153)\end{array}$ & $\begin{array}{l}-0.093 \\
(0.233)\end{array}$ \\
\hline Urbanization & $\begin{array}{l}-0.045 \\
(0.133)\end{array}$ & $\begin{array}{l}-0.031 \\
(0.172)\end{array}$ & $\begin{array}{l}0.077 \\
(0.056)\end{array}$ & $\begin{array}{l}0.071 \\
(0.072)\end{array}$ \\
\hline Intercept & $\begin{array}{l}6.236 \\
(10.20)\end{array}$ & $\begin{array}{l}5.775 \\
(13.25)\end{array}$ & $\begin{array}{l}-4.163 \\
(4.271)\end{array}$ & $\begin{array}{l}-3.975 \\
(5.558)\end{array}$ \\
\hline R-squared & 0.964 & 0.965 & 0.998 & 0.998 \\
\hline
\end{tabular}

*** significance @ 1\% level, ** significance @ 5\% level, * significance @ $10 \%$ level. Numbers in parenthesis are standard errors.
TABLE V: RESULTS OF FIXED EFFECTS AND RANDOM EFFECTS FOR NON-ECONOMIC VARIABLES

\begin{tabular}{|c|c|c|c|c|}
\hline \multirow[t]{2}{*}{$\begin{array}{c}\text { Non-Economic } \\
\text { Variables }\end{array}$} & \multicolumn{2}{|c|}{$\begin{array}{c}\text { Economically Active } \\
\text { Population }\end{array}$} & \multicolumn{2}{|c|}{ Sixty Plus Years Old } \\
\hline & $\mathrm{RE}$ & $\mathrm{FE}$ & $\mathrm{RE}$ & $\mathrm{FE}$ \\
\hline Union Density & $\begin{array}{l}-0.050 \\
(0.587)\end{array}$ & $\begin{array}{l}-0.058 \\
(0.619)\end{array}$ & $\begin{array}{l}-0.066 \\
(0.156)\end{array}$ & $\begin{array}{l}-0.069 \\
(0.156)\end{array}$ \\
\hline SS Benefits & omitted & omitted & omitted & omitted \\
\hline Feminist Theory & $\begin{array}{c}0.002 \\
(0.740)\end{array}$ & $\begin{array}{c}-0.027 \\
(0.782)\end{array}$ & $\begin{array}{c}0.377 \\
(0.196)^{*}\end{array}$ & $\begin{array}{c}0.364 \\
(0.197)\end{array}$ \\
\hline Education & $\begin{array}{c}0.212 \\
(0.497)\end{array}$ & $\begin{array}{c}0.254 \\
(0.529)\end{array}$ & $\begin{array}{c}0.596 \\
(0.132)^{* * *}\end{array}$ & $\begin{array}{c}0.615 \\
(0.133)^{* * * *}\end{array}$ \\
\hline Informal Sectors & $\begin{array}{l}-0.353 \\
(0.352)\end{array}$ & $\begin{array}{l}-0.319 \\
(0.376)\end{array}$ & $\begin{array}{c}-0.165 \\
(0.093)^{*}\end{array}$ & $\begin{array}{l}-0.149 \\
(0.095)\end{array}$ \\
\hline Family Obligation & $\begin{array}{l}-1.314 \\
(1.125)\end{array}$ & $\begin{array}{l}-4.500 \\
(1.230\end{array}$ & $0.408(0.299)$ & $\begin{array}{c}0.325 \\
(0.310)\end{array}$ \\
\hline Poverty Incidence & $\begin{array}{l}-0.280 \\
(0.968)\end{array}$ & $\begin{array}{l}-0.335 \\
(1.024)\end{array}$ & $\begin{array}{c}0.470 \\
(0.257)^{*}\end{array}$ & $\begin{array}{c}0.445 \\
(0.258)\end{array}$ \\
\hline Intercept & $\begin{array}{c}59.76 \\
(49.30)\end{array}$ & $\begin{array}{c}61.64 \\
(52.05)\end{array}$ & $\begin{array}{c}-40.40 \\
(13.07)^{* * *}\end{array}$ & $\begin{array}{c}-39.55 \\
(13.11)^{* *}\end{array}$ \\
\hline R-squared & 0.766 & 0.767 & 0.994 & 0.994 \\
\hline
\end{tabular}

*** significance @ 1\% level, ** significance @ 5\% level, * significance @ $10 \%$ level. Numbers in parenthesis are standard errors.

To choose between fixed and random effects models, the Haussmann specification test was conducted. Results showed that the null hypothesis "the random effects model is more consistent in estimating social insurance coverage" is not rejected whether for the economically active population or for the elderly.

The models were also regressed using the GMM results of which are shown in Table VI with economic variables and in Table VII with non-economic variables.

TABLE VI: RESUlTS OF GENERALIZED METHOD OF MOMENTS FOR ECONOMIC VARIABLES

\begin{tabular}{lllll}
\hline \hline Economic Variables & \multicolumn{2}{c}{$\begin{array}{c}\text { Economically Active } \\
\text { Population }\end{array}$} & \multicolumn{1}{c}{ Sixty Plus Years Old } \\
\hline $\begin{array}{lllll}\text { Economic } \\
\text { Development }\end{array}$ & 30.81 & 13.44 & -22.17 & $4.451^{* *}$ \\
Modernization & -30.60 & 13.30 & 21.97 & $4.400^{* *}$ \\
Economic Stability & 0.540 & 0.525 & -1.174 & $0.175^{* *}$ \\
Capital Formation & 0.164 & $0.047^{*}$ & -0.082 & $0.015^{* *}$ \\
Admin Capacity & -0.921 & 0.436 & 1.427 & $0.148^{* *}$ \\
Wage Growth & -0.136 & 0.207 & 0.960 & $0.070^{* * *}$ \\
Globalization & -0.623 & $0.177^{*}$ & 0.097 & 0.061 \\
Global Crisis & -8.082 & 3.577 & 8.788 & $1.186^{* *}$ \\
Unemployment & -0.295 & 0.397 & 0.298 & 0.134 \\
Underemployment & -0.046 & 0.311 & 0.347 & $0.107^{*}$ \\
Urbanization & -0.622 & 0.227 & 0.426 & $0.075^{* *}$ \\
Intercept & 90.20 & 32.12 & -59.12 & $10.77^{* *}$ \\
R-squared & 0.986 & & 0.999 & \\
\hline
\end{tabular}

*** significance @ 1\% level, ** significance @ 5\% level, * significance @ $10 \%$ level 
After presenting the results of the four models of multiple regression analysis, it is now imperative to recapitulate the effects of the different variables on social insurance coverage. Only those variables with standard errors nearing zero $(\leq 0.3)$ and are significant were considered determinants of social insurance coverage as reflected in Table VIII.

TABLE VII: RESULTS OF GENERALIZED METHOD OF MOMENTS FOR NON-ECONOMIC VARIABLES

\begin{tabular}{lcccc}
\hline \hline & \multicolumn{2}{c}{$\begin{array}{c}\text { Economically Active } \\
\text { Population }\end{array}$} & Sixty Plus Years Old \\
\cline { 2 - 5 } \multicolumn{1}{c}{ Variables } & Coefficient & $\begin{array}{c}\text { Standard } \\
\text { Errors }\end{array}$ & Coefficient & $\begin{array}{l}\text { Standard } \\
\text { Errors }\end{array}$ \\
\cline { 2 - 5 } Union Density & -0.008 & 0.526 & 0.044 & 0.122 \\
$\begin{array}{l}\text { SS Benefits } \\
\text { Feminist }\end{array}$ & -0.928 & 3.256 & 0.266 & 0.944 \\
$\begin{array}{l}\text { Theory } \\
\text { Education }\end{array}$ & -0.414 & 1.039 & 0.401 & 0.297 \\
$\begin{array}{l}\text { Informal } \\
\text { Sectors }\end{array}$ & -0.231 & 0.694 & 0.698 & $0.1916^{* *}$ \\
$\begin{array}{l}\text { Family } \\
\text { Obligation }\end{array}$ & -1.918 & 1.377 & 0.297 & 0.272 \\
$\begin{array}{l}\text { Poverty } \\
\text { Incidence }\end{array}$ & 0.91 & 0.863 & 0.604 & $0.231^{* *}$ \\
Intercept & 73.7 & 42.56 & -48.69 & $11.07^{* * *}$ \\
R-squared & 0.8 & & 0.997 & \\
\hline \hline
\end{tabular}

*** significance @ 1\% level, ** significance @ 5\% level, * significance @ $10 \%$ level.

TABLE VIII: RECAPITULATION OF THE RESULTS OF THE DIFFERENT REGRESSION MODELS

\begin{tabular}{|c|c|c|c|c|c|c|c|c|c|c|}
\hline \multirow[t]{2}{*}{ Variables } & \multicolumn{5}{|c|}{ Economically Active Population } & \multicolumn{5}{|c|}{ Sixty Plus Years Old } \\
\hline & GLM & OLS & $\mathrm{RE}$ & GMM & SUM & GLM & OLS & RE & GMM & SUM \\
\hline \multicolumn{11}{|l|}{ Economic } \\
\hline $\begin{array}{l}\text { Economic } \\
\text { Development }\end{array}$ & & & -2 & & - & & & $\checkmark$ & & + \\
\hline \multicolumn{11}{|l|}{ Modernization } \\
\hline $\begin{array}{l}\text { Economic } \\
\text { Stability }\end{array}$ & & & $\checkmark$ & & + & $\checkmark$ & $\checkmark$ & $\checkmark$ & $\checkmark$ & + \\
\hline Capital Formation & $\checkmark$ & $\checkmark$ & $\checkmark$ & $\checkmark$ & + & -2 & -2 & & -2 & - \\
\hline $\begin{array}{l}\text { Administrative } \\
\text { Capacity }\end{array}$ & & & & & & $\checkmark$ & $\checkmark$ & $\checkmark$ & $\checkmark$ & + \\
\hline Wage Growth & & & $\checkmark$ & & + & $\checkmark$ & $\checkmark$ & $\checkmark$ & $\checkmark$ & + \\
\hline Globalization & -2 & & & $-\sqrt{ }$ & - & & & & & \\
\hline \multicolumn{11}{|l|}{ Global Crisis } \\
\hline Unemployment & & & & & & $\checkmark$ & & & & + \\
\hline $\begin{array}{l}\text { Under } \\
\text { employment }\end{array}$ & & & & & & $\checkmark$ & & & $\checkmark$ & + \\
\hline Urbanization & $\checkmark$ & & & & + & -2 & $-\checkmark$ & & -2 & - \\
\hline \multicolumn{11}{|l|}{ Non-economic } \\
\hline \multicolumn{11}{|l|}{ Union Density } \\
\hline \multicolumn{11}{|l|}{$\begin{array}{l}\text { Social Security } \\
\text { Benefits }\end{array}$} \\
\hline Feminist Theory & & & & & & $\checkmark$ & & $\checkmark$ & & + \\
\hline Education & & & & & & $\checkmark$ & $\checkmark$ & $\checkmark$ & $\checkmark$ & + \\
\hline Informal Sectors & & & & & & -2 & & -2 & & - \\
\hline \multicolumn{11}{|l|}{ Family Obligation } \\
\hline Poverty Incidence & & & & & & $\checkmark$ & $\checkmark$ & $\checkmark$ & $\checkmark$ & + \\
\hline
\end{tabular}

From the table, it is evident that a few of the hypotheses proposed in the study were rejected but some were supported. For the dependent variable EAP, only economic factors were statistically chosen as determinants of coverage namely: economic stability, capital formation, wage growth, urbanization, economic development and globalization. Note that the last 2 variables have negative impact on EAP. While for the dependent variable $60+$, the following are the economic drivers: economic development, economic stability, capital formation, administrative capacity, wage growth, unemployment, underemployment and urbanization, with only capital formation and urbanization showing negative impacts. The following non-economic variables positively influenced coverage among the elderly: feminist theory, education and poverty incidence, while the factor informal sector, negatively influenced the elderly coverage.

\section{Discussions}

Testing the correlations between social insurance coverage as a dependent variable (DV) and the different economic and non-economic factors as the independent variables (IVs), has led to obtaining results that give evidence to the influence of some IVs on the DV. The analysis develops around four multiple regressions showing the indicators of social insurance coverage as either the contributors among the economically active population (EAP) or the elderly beneficiaries $(60+)$.

With EAP as the proxy for coverage, only some economic factors were found to drive it. EAP is negatively associated with economic development which supports the contention of ILO [5] that at the macro level, there does not seem to be a clear relationship between social security and development. Further, the negative result in this study also substantiates the argument of ILO [7] that the aforementioned DV and IV are reversed; social security is one of the conditions of economic development. The positive relationship between EAP and economic stability and that between EAP and capital formation are in line with the findings of Hong [12] stated as "an increased capacity in national accounts possibly impact public welfare expenditures". However, with respect to globalization, it nullify the findings of Hong that globalization did not successfully predict variability in his dependent variable, economic crisis did. The negative impression of globalization in the present study seems more acceptable because it is premised on the idea that globalization exposes societies to greater income insecurity. The study's positive effect of wage growth and urbanization affirms the conclusion of Auerbach, et. al [36] that workers with low wages and workers in rural area find social security programs less attractive. Conversely, Rofman and Oliveri [4] and Gao et. al [35] found that manufacturing and service employees have better coverage rates. This also confirms the theory of Hong [12] that decreasing employment levels in agriculture are helpful in predicting increasing levels of social expenditures.

More IVs qualified as determinants of coverage in this study when the DV is the elderly beneficiaries (60+). Aside from the factors already discussed in EAP, other economic and non-economic variables impact elderly coverage exclusive of globalization. Economic development here is found to have a positive effect on coverage unlike in EAP. This may support the position of ILO [5] that at the sectorial and enterprise levels, there is a good reason to believe that there is a positive relation between development and social security. There are also reversed findings with regards the force of capital formation and urbanization on coverage. However, the positive relation of administrative capacity reaffirms the earlier statement of Hong [12] that increased capacity in national accounts positively impact public welfare expenditures. With regards the positive influence of unemployment and underemployment, the findings boost the declaration "the amount paid out in disability pensions falls immediately when unemployment reduces" and can be 
explained further by "more people with reduced working capacity choose to return to work and pay social charges". It negates however, the pronouncement of Mesa-Lago et. al [6] "high unemployment and underemployment rates are reasons for low coverage in the Philippines" and by Auerbach et. al [36]: "part-time work may be a form of evasion".

The non-economic variable, feminist theory has a positive relation with the $60+$ coverage in accordance with the judgment of Rofman and Oliveri [4]: "women usually have lower coverage rates than men, especially among the elderly". The value of education as a determinant of coverage is confirmed by Rickne [22] and Corripio [8]. The negative influence of informal sectors is in agreement with the findings of Mesa-Lago et al. [6], Kaseke [2] and ILO [7]. The last variable that has a positive impact on coverage is poverty incidence. This is not in accordance with the negative association found by Mesa-Lago et al. and ILO. However, Rofman and Oliveri declared that in the 2000s, trend seems to have reversed, thus confirming the findings of this study that poorer workers have more participation in contributory pension systems.

\section{CONCLUSION}

This study analyzed the coverage of the most dominant form of social security which is social insurance or public pension in the Philippines by examining its relationship with selected key economic and non-economic variables. It presents indicators of social insurance coverage for the period 2000-2013 including pension coverage among the economically active population (EAP) and the elderly beneficiaries $(60+)$. Some conclusions became evident in this study, as follows:

1) Social insurance coverage represented by the contributors from the EAP is positively influenced by the following economic factors: economic stability, capital formation, wage growth and urbanization; and negatively affected by the degree of economic development and globalization.

2) Social insurance coverage indicated by the percentage of population 60 years or more who receive pension benefits is positively induced by the following economic variables: economic development, economic stability, national administrative capacity, wage growth, unemployment and underemployment; and negatively pushed by capital formation and urbanization.

3) Coverage among the $60+$ beneficiaries is positively driven by the following non-economic factors: feminist theory, education and poverty incidence; while informal sector has negative impression on it.

4) More statistically significant determinants for the 60+ beneficiaries confirms the conclusion of Rofman and Oliveri [4] that assessing coverage among the elderly presents fewer difficulties since instead of measuring the accumulation of potential rights, the focus is on the proportion of eligible individuals who are currently receiving benefits.

Knowledge from this study may contribute to policy-making decisions by the Philippine government. However, results do not represent a complete analytical framework to explain the determinants of social insurance coverage. Yet, they provide a leading set of statistical data from which future works with improvements could raise some implications for public policy such as: using the total amount of social security expenditure (measured as a ratio of GDP or of total spending) as a proxy aggregate indicator of coverage. In addition, the concept of economic development must be taken on the context of a people-centered development because this seems to be more related to decent work embracing social security. Lastly, future work should employ other rigorous statistical methods to complement the results of this study.

\section{REFERENCES}

[1] International Labor Office (ILO). (2000). World Labor Report. International Labor Office, Geneva. [Online]. Available: www.ilo.org/publns

[2] E. Kaseke. (2000). The challenges of extending social security to the poor: An African perspective. [Online]. Available: http://www. users/s670/downloads/ICWS.html

[3] A. King. Determinants of employees' participation and contribution to the Philippine SSS. [Online]. Available: www.arnoldking.com/econ/saving/socsec

[4] R. Rofman and M. L. Oliveri, "Pension coverage in Latin America. Trends and determinants," Social Protection and Labor, The World Bank, 2012.

[5] International Labor Office (ILO). (2001). Social security: Issues, challenges and prospects. [Online]. Available: www.ilo.org/publns

[6] M. Lago, V. O. Viajar, and R. Castillo. (2011). Pension in the Philippines: Challenges and ways forward. Friedrich-Ebert-Stiftung Office. [Online]. Available: www.fes.org.ph

[7] International Labor Office (ILO). (2010-2011). World Social Security Report: Provoking coverage in times of crisis and beyond. International Labor Office, Geneva. [Online]. Available: www.ilo.org/publns

[8] E. F .Corripio. (2012). Creating incentives for voluntary contributions to pension funds by independent workers; An informal evaluation based on the case of Chile. [Online]. Available: https://www.bbvaresearch.com/wp.../WP_1012_tcm348-221423.pdf

[9] S. Levy, Good Intentions, Bad Outcomes: Social Policy, Informality and Economic Growth in Mexico, The Brookings Institute, Washington, D.C.

[10] S. Galiani and F. Weinschelbaum, "Modelling informality: Households and firms," Economic Inquiry, vol. 50, no. 3, pp. 821-838, 2012.

[11] S. Kitao, "A life-cycle model of unemployment and disability insurance," Journal of Monetary Economics, no. 68, pp. 1-18, 2014.

[12] I. Hong, "Trade and determinants of social expenditures in Korea, Japan and Taiwan," Social Policy and Administration, vol. 48, no. 6, pp. 647-665, 2014.

[13] Y. M. Kim, "Beyond East Asia welfare productivist in South Korea," Policy and Politics, vol. 36, no. 1, pp. 109-125, 2008.

[14] W. I. Lin, "The structural determinants of welfare effort in post-war Taiwan," International Social Work, no. 34, pp. 171-190, 1991.

[15] M. H. Kim, and J. K. Jung, "An empirical study on changing factors of social welfare expenditures of Korea," Korea Social Security Research, vol. 19, no. 1, pp. 1-21, 2003.

[16] S. H. Ahn and S. H. Baek, "A time series study on the determinants behind the changes of Korea welfare state," Korean Journal of Social Welfare Studies, no. 37, pp. 117-144, 2008.

[17] K. Rupp and D. Stapleton, "Determinants of growth in social security administators' disability programs - An overview," Social Security Bulletin, vol. 58, no. 4, pp. 43-53, 1995.

[18] S. H. Ahn and S. S. Lee, "Explaining Korea welfare state development with new empirical data and methods," Asian Social Work and Policy Review, no. 6, pp. 67-85, 2012.

[19] C. Park, "The divergent paths of East Asia welfare regimes: The effects of production regimes and democratization," Korean Social Science Journal, vol. 24, no. 1, pp. 39-70, 2007.

[20] A. Croisant, "Changing welfare regimes in East and Southeast Asia: Crisis, change and challenge," Social Policy and Administration, vol. 38, no. 5, pp. 504-524, 2014.

[21] M. Cristea, N. Marcu, and S. Carstina, "The relationship between insurance and economic growth in Romania compared to the main results in Europe - A theoretical and empirical analysis," Procedia Economics and Finance, no. 8, pp. 226-235, 2014. 
[22] J. Rickne, "Labor market conditions and social insurance in China," China Economic Review, no. 27, pp. 52-68, 2013.

[23] F. Cai, "Demographic transition, demographic dividend, and Lewis turning point in China," Economic Research Journal, no. 4, pp. 107-119, 2010

[24] X. Meng, "Labor market outcomes and reforms in China," Journal of Economic Perspectives, no. 24, pp. 75-101, 2012.

[25] B. Blechova, J. Janouskova, and S. Sobotovicova, "Seniors' position in the labor market from the point of view of income and related insurance and health contributions," Procedia - Social and Behavioral Science, no. 110, pp. 1042-1051, 2014.

[26] Y. Lu, Z. Tao, and Y. Wang, "Union effects on performance and employment relations: Evidence from China," China Economic Review, no. 21, pp. 202-210, 2010.

[27] C. Nyland, R. Smyth, and C. J. Zhu, "What determines the extent to which employers will comply with their social security obligations? Evidence from Chinese firm level data," Social Policy Administration, no. 40, pp. 196-214, 2006.

[28] I. Nielsen and R. Smith, "Who bears the burden of employer compliance with social security contributions? Evidence from Chinese firm level data," China Economic Review, no. 19, pp. 230-244, 2007.

[29] J. Mao, L. Zhang, and J. Zhao. Social security, taxation and compliance: The Chinese evidence. [Online]. Available: http://ssrn.com/abstract=2151695

[30] C. Nyland, B. S. Thomson, and C. J. Zhu, "Employee attitudes toward social insurance compliance in Shanghai, China," International Social Security Review, no. 64, pp. 73-98, 2011.

[31] Z. Cheng, I. Nielsen, and R. Smith. (2013). Access to social insurance in urban China: A comparative study of rural-urban and urban-urban migrants in Beijing. [Online]. Available: www.sciencedirect.com/science/article/pii/S019739751300091X

[32] F. Guo and W. S. Gao, "What determines the welfare and social security entitlements of rural migrants in Chinese cities," Migration and Social Protection in China, I. Nielsen and R. Smith, Ed.

[33] R. Smyth, I. Nielsen, and X. Qian, "What determines employer willingness to "top up" social insurance? Evidence from Shanghai's 25 plus x scheme," International Journal of Manpower, no. 30, pp. 512-528, 2009.

[34] I. Nielsen, C. Nyland, R. Smith, M. Zhang, and C. Zhu, "Which rural migrants receive social insurance in Chinese cities?" Global social Policy, no. 5, pp. 353-381.

[35] Q. Gao, S. Yang, and S. Li, "Labor contracts and social insurance participation among migrant workers in China," China Economic Review, no. 23, pp. 1195-1205, 2012.

[36] P. Auerback, M. E. Genoni, and C. Pages. (2007). Social security coverage and the labor market in developing countries. The Institute for the Study of Labor (IZA), Bonn. Discussion Paper No. 2979. [Online] Available: www.iadb.org/res/publications/pubfiles/pubWP-537.pdf

[37] J. Khan, U. Gerdtham, and B. Jansson, "Effects of macroeconomic trends on social security spending due to sickness and disability," American Journal of Public Health, vol. 94, no. 11, 2004.

[38] J. Turner, "Retirement income system for different economic, demographic and political environments," OECD Working Paper AWP 3.8, 1998.

[39] C. Aspalter. (2006). The East Asia welfare regime: A political-cultural perspective. [Online]. Available: http://www.welfareasia.org/2ndconference/paper/Christian\%20Aspalt er_Paper(2ndEASP).doc.

[40] Y. W. Ku and C. J. Finer, "Developments in East Asia welfare studies," Social Policy and Administration, vol. 41, no. 2, pp. 115-131, 2007.

[41] C. Jensen, "Determinants of welfare service provision after the golden age," International Journal of Social welfare, no. 20, pp. 125-134, 2011.

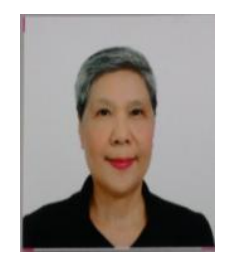

Ma. Belinda S. Mandigma was born in Batangas City, Philippines on July 15, 1953. She finished her P.h.D. in commerce from the University of Santo Tomas, Manila, Philippines in 1995. She also finished a graduate course for CFOs from the Asian Institute of Management, Makati, Philippines in 2014. At present, she is a professor at the Graduate School and at the College of Commerce \& Business Administration of the University of Santo Tomas and a research associate at the University's Research center on culture, education and social issues. She is also a research fellow of the World Business Institute, Melbourne, Australia and an associate editor of the World Journal of Social Sciences, Zia World Press in Australia. Her research interests are in the field of finance, accounting, management and social science.

Prof. Mandigma is a senior member of the International Economic Development Research Center (IEDRC), a regular member of the Chartered Financial Analyst (CFA) Charlottesville, VA, USA and a CFA level II passer. She was awarded best paper presenter at the International Conference on Business Strategy and Social Sciences (ICBSSS 2014) held on December 12-13, 2014 in Jakarta, Indonesia and was given a best paper certificate at the 2nd International Conference on Information and Finance (ICIF 2012), held on November 24-25, 2012 in Bangkok, Thailand. She is also a technical committee of the International Postgraduate Network, Selangor, Malaysia. 